www.nature.com/jhg

\title{
The regulation-of-autophagy pathway may influence Chinese stature variation: evidence from elder adults
}

\author{
Feng Pan ${ }^{1,5}$, Xiao-Gang Liu ${ }^{1,5}$, Yan-Fang Guo ${ }^{1}$, Yuan Chen ${ }^{1}$, Shan-Shan Dong ${ }^{1,2}$, Chuan Qiu ${ }^{1}$, Zhi-Xin Zhang ${ }^{1}$, \\ Qi Zhou ${ }^{1}$, Tie-Lin Yang ${ }^{1,2}$, Yan Guo ${ }^{1,2}$, Xue-Zhen Zhu ${ }^{1}$, Hong-Wen Deng ${ }^{1,2,3,4}$
}

Recent success of genome-wide association studies (GWASs) on human height variation emphasized the effects of individual loci or genes. In this study, we used a developed pathway-based approach to further test biological pathways for potential association with stature, by examining $\sim 370000$ single-nucleotide polymorphisms (SNPs) across the human genome in 618 unrelated elder Han Chinese. A total of 626 biological pathways annotated by any of the three major public pathway databases (KEGG, BioCarta and Ambion GeneAssist Pathway Atlas) were tested. The regulation-of-autophagy (ROA) (nominal $P=0.012$ ) pathway was marginally significantly associated with human stature after our family wise error rate multiple-testing correction. We also used 1000 random recruited US whites for further replication. Interestingly, the ROA pathway presented the strongest signals in whites for height variation (nominal $P=0.002$ ). The results correspond to biological roles of the ROA pathway in human long bone development and growth. Our findings also implied that multiple-genetic factors may work jointly as a functional unit (pathway), and the traditional GWASs could have missed important genetic information imbedded in those less significant markers.

Journal of Human Genetics (2010) 55, 441-447; doi:10.1038/jhg.2010.44; published online 7 May 2010

Keywords: autophagy; GWAS; height; pathway; stature

\section{INTRODUCTION}

Human stature has a long research history in the community, as it is the most visible traits with remarkable high heritability and can be easily and accurately measured. ${ }^{1}$ Specifically in Han Chinese, heritability of stature is $>0.6 .^{2}$ The major driver of those studies on stature is to provide clues for human growth, bone development and onset of some diseases such as cancer, type 2 diabetes, $\mathrm{H}$ syndrome and osteoporosis. ${ }^{1,3,4}$

Recent success in genome-wide association studies (GWASs) has revealed that some pathways can influence the human height. ${ }^{5-10}$ Strong statistical and experimental evidence showed that pathways underlying bone and cartilage development have critical roles in the formation of adult height, ${ }^{1}$ such as pathways of Hedgehog signaling, ${ }^{6}$ bone morphogenic ${ }^{9,11}$ and extracellular matrix. ${ }^{1}$ Meanwhile, some pathways may have indirect impact on human height and highlight more fundamental biological processes, including the pathways of chromatin structure regulation, ${ }^{6}$ cell-cycle regulation ${ }^{1}$ and the C-type natriuretic peptide signaling. ${ }^{12}$

Our earlier GWAS performed in Han Chinese ${ }^{13}$ pinpointed some single-nucleotide polymorphisms (SNPs) that was influencing Chinese height variation. Nevertheless, the most significant SNPs can only explain limited variance $(\sim 0.3 \%)$ in stature. ${ }^{13}$ The pathway-based association test integrates current knowledge about functional interactions among genes and represents a promising approach to look into genome-wide data to find the potential joint effects or functional interactions of genes, which were imbedded in the remaining unreported SNPs that did not achieve the genome-wide significant level. ${ }^{14}$

In this study, the genome-wide pathway-based analysis was conducted to detect the potential association between 626 pathways and height variation in Han Chinese. We aimed to identify pathways that can be influencing Chinese stature and/or to provide novel hypotheses in the mechanisms of human growth. This study may provide new insights into height variation, especially for the Chinese ethnic, which was less studied and genetically differs from the whites. ${ }^{1,9,13}$

\section{MATERIALS AND METHODS}

\section{Subjects}

The study was approved by the Research Administration of Xi'an Jiaotong University to assure that the general ethnic rules were addressed. All study volunteers have signed the informed-consent forms before they entered the

\footnotetext{
${ }^{1}$ The Key Laboratory of Biomedical Information Engineering of Ministry of Education, and Institute of Molecular Genetics, School of Life Science and Technology, Xi'an Jiaotong University, Xi'an, Shaanxi, China; ${ }^{2}$ Departments of Orthopedic Surgery and Basic Medical Science, School of Medicine, University of Missouri-Kansas City, Kansas City, MO, USA; ${ }^{3}$ College of Life Sciences and Engineering, Beijing Jiao Tong University, Beijing, PR China and ${ }^{4}$ Center of Systematic Biomedical Research, Shanghai University of Science and Technology, Shanghai, PR China

${ }^{5}$ These authors contributed equally to this work.

Correspondence: Dr H-W Deng, Departments of Orthopedic Surgery and Basic Medical Sciences, University of Missouri-Kansas City, 2411 Holmes Street, Room M3-C03, Kansas City, MO 64108, USA.

E-mail: dengh@umkc.edu

Received 19 January 2010; revised 3 April 2010; accepted 7 April 2010; published online 7 May 2010
} 
study. A total of 618 elder Han adults were randomly recruited from the Xi'an City and its surrounding areas in northern China. Basic characteristics of the study sample are given in Table 1a. Stature for each subject was measured using a stadiometer wearing no shoes. The daily calibrated stadiometer was in well maintenance and the long-term precision was monitored. The coefficient of variation of stature measurements was $5.8 \%$. Strict data quality control was conducted by excluding the outliers and database inputting errors. The whole data set was double crosschecked by at least two different researchers.

All the individuals with chronic diseases and conditions that might potentially affect hormone level, bone structure or metabolism were excluded from this study, which included but not limited to chronic disorders involving vital organs, diabetes, hyperthyroidism, hypo-/hyper-parathyroidism and other serious skeletal diseases (Paget's disease, osteogenesis imperfecta, rheumatoid arthritis, kyphosis, serious vertebral fracture), chronic use of drugs affecting bone metabolism (hormone replacement therapy, corticosteroid therapy, anticonvulsant drugs, anti-bone-resorptive or bone anabolic agents) and malnutrition conditions (such as anorexia nervosa).

\section{Genotyping}

Genomic DNA was extracted from whole human blood using a commercial isolation kit (Gentra systems, Minneapolis, MN, USA) following the standard protocols. For each sample, genotyping with GeneChip Human Mapping $500 \mathrm{~K}$ set containing $250 \mathrm{~K} \mathrm{Nsp}$ array and $250 \mathrm{~K}$ Sty array (Affymetrix, Santa Clara, CA, USA) was performed using the standard protocols recommended by the manufacturer.

From the initial full-set of 500568 SNPs, we discarded 18067 SNPs with sample call rate $<90 \%, 13041$ SNPs with allele frequencies extremely deviating from Hardy-Weinberg equilibrium $\left(P<10^{-7}\right)$ and 98202 SNPs with minor allele frequencies $<1 \%$. The final SNP number for the analyses is 371258 . This SNP set covered 14642 genes and yielded an average marker spacing of $\sim 7.9 \mathrm{~kb}$ throughout the human genome.

\section{Population stratification}

We used STRUCTURE 2.2 program (http://pritch.bsd.uchicago.edu/software.html) to detect the potential population stratification of our sample. The program used a Markov chain Monte Carlo algorithm to cluster individuals into cryptic subpopulations on the basis of multi-loci genotype data. ${ }^{15}$ We performed the analysis assuming the number of population strata $k=2$ and using a set of 1000 unlinked SNPs randomly selected genome wide. EIGENSTRAT ${ }^{16}$ was used to crosscheck the stratification in our analyses.

\section{Generation of pathway/gene sets collection}

To identify the pathways potentially contributing to Chinese stature variation, we comprehensively retrieved the pathways collected by three major pathway databases, KEGG (http://www.genome.ad.jp/kegg/pathway.html), BioCarta (http://www.biocarta.com/genes/index.asp) and Ambion GeneAssist Pathway Atlas (http://www.ambion.com/tools/pathway). The number of pathways finally retrieved from BioCarta, KEGG and Ambion were 364, 262 and 201, respectively. To relieve the multiple-testing issue and avoid testing over narrow or broad functional pathways, we only included those pathways with 10-200 genes and at least $85 \%$ of which were covered by at least one SNP of our qualified genotypic data. The pathways with the following characteristics were filtered: (1) the pathway contains $<10$ or $>200$ genes in the above three database; (2) after discarding those unqualified SNPs (bad sample call rate, no HardyWeinberg equilibrium, small minor allele frequencies), the pathway contains $<10$ or $>200$ genes in our genotypic data; (3) after filtering those SNPs that were $500 \mathrm{~kb}$ away from the involved gene, the pathway contains $<10$ or $>200$ genes in our cleaned data and (4) the pathway contains genes within 10-200 gene, but $<85 \%$ of them were included in our analysis due to discarded SNPs or SNPs with $>500 \mathrm{~kb}$ distance. Overall, a total of 626 pathways/functional networks were finally analyzed.

\section{Pathway-based GWAS analysis}

Gender and age are significant fixed effectors on stature ${ }^{13}$ and they were used to adjust the raw stature data. The GWAS analysis for individual SNPs was achieved by the Wald $t$-test implemented in Plink (version 1.03). ${ }^{17}$
Table 1a Basic characteristics of the 618 studied Chinese (mean \pm s.d.)

\begin{tabular}{lrcr}
\hline Trait & Total $(\mathrm{n}=618)$ & Female $(\mathrm{n}=351)$ & Male $(\mathrm{n}=267)$ \\
\hline Age (years) & $70.6 \pm 7.8$ & $70.2 \pm 8.2$ & $71.1 \pm 7.1$ \\
Stature $(\mathrm{cm})$ & $160.9 \pm 9.0$ & $155.5 \pm 6.2$ & $168.0 \pm 6.9$ \\
\hline
\end{tabular}

Table 1b Basic characteristics of the 1000 studied American whites (mean \pm s.d.)

\begin{tabular}{lccr}
\hline Trait & Total $(\mathrm{n}=1000)$ & Female $(\mathrm{n}=501)$ & Male $(\mathrm{n}=499)$ \\
\hline Age (years) & $50.3 \pm 18.3$ & $50.1 \pm 7.7$ & $50.5 \pm 8.9$ \\
Stature $(\mathrm{cm})$ & $170.8 \pm 9.8$ & $163.8 \pm 6.5$ & $177.8 \pm 7.0$ \\
\hline
\end{tabular}

The used pathway-based GWAS approach was proposed and developed first by Wang et al. ${ }^{14}$

SNPs that are $500 \mathrm{~kb}$ away from any gene are not considered. The SNPs of our identified pathway were verified without overlapping or misrepresentation problem (Figure 2). Among all the SNPs of a given gene, the SNP achieving the smallest $P$-value (the highest Wald $t$-test statistic values) in the single-marker association tests was assigned to represent the magnitude of association evidence of the gene. If in very rare cases where one SNP is located within shared regions of two overlapping genes, we map the SNP to both genes. They will all get the same score. We ranked all genes by sorting their statistic values from the largest to smallest. Then, a weighted Kolmogorov-Smirnov-like running-sum enrichment score (ES) was calculated. ${ }^{14}$ Briefly, ES score was used to reflect the enrichment of a given gene set at the top of the entire genome-wide ranked gene list. It measures the deviation from zero to maximum level of the highest statistic value encountered in the random walk. High ES score means this set of genes (genes of pathway) concentrated at the top of ranked gene list.

The nominal significance level of ES for each pathway was estimated by shuffling phenotypic data. The procedures of ES calculation were repeated for 1000 times. Finally, an empirical distribution of ES (ES $\left.{ }_{\text {null }}\right)$ was generated for each pathway. The nominal significance of an observed ES for a pathway (nominal $P$-value) was estimated as the percentage of permutations whose $\mathrm{ES}_{\text {null }}>$ observed ES.

Finally, two measures were used to adjust for multiple-hypothesis testing for more reliable results. We first normalized the ES to correct the influence of gene size, so that different gene sets are directly comparable to each other. The falsediscovery rate (FDR) $Q$ value (denoted as $Q_{\mathrm{FDR}}$ ) and family wise error rate (FWER) $P$-value (denoted as $P_{\mathrm{FWER}}$ ) were generated for correcting the multiple testing. ${ }^{14}$ FDR is a procedure that is usually used to control the fraction of expected false-positive findings to stay below a certain threshold. FWER is another approach that is considered to be highly conservative seeking to ensure that the list of reported results does not include even a single falsepositive gene set.

To identify the common pathway(s) that may influence height in distinct ethnics, and also as a replication effort, we compared the results of this study with those of the pathway-based GWAS analysis for height variation in our American white sample study, which was approved by the University of Missouri-Kansas City Institutional Review Board. A total of 1000 whites were randomly recruited from Midwestern of United States (Table 1b). The same genotyping and statistical approaches were applied across the Chinese and the American studies. 5

\section{RESULTS}

\section{Characteristics of study subjects}

A total of 618 individuals were included in the analysis. Basic characteristics of the study sample are shown in Table 1a. 
The STRUCTURE program results showed that all the subjects were clustered together as a single group by using 1000 unlinked SNPs, which suggested that no significant population stratification was present in our sample. The analyses by EIGENSTRAT confirmed that only one principal component was significant $(P<0.001)$, which suggested that our study sample originated from one common ancestry population. Consequently, the inflation factor $\lambda$ calculated by the Genomic Control approach ${ }^{18}$ implemented in EIGENSTRAT is 1.02 , close to 1 standing for no stratification.

Pathway-based association analysis

Out of the 626 pathways, the regulation-of-autophagy (ROA) pathway achieved the strongest evidence of association with height variation (nominal $P=0.012, Q_{\mathrm{FDR}}=0.30$ and $P_{\mathrm{FWER}}=0.11$ ). The ROA pathway was involved in 35 genes (data from KEGG database), with 28 genes covered by our SNP set (Table 2). Among the 28 genes, 5 genes (PIK3C3, ULK2, ATG5, GABARAPL1 and IFNG) got nominal $P<0.01$, and 14 genes were ranked among the top 5000 (leading edge) genes in the single-SNP GWAS. The ROA pathway achieved a high ES score of 0.47 with nominal $P$-value $=0.012$ (Figure 1). The individual gene with the most significant $P$-value among all the 28 genes is PIK3C3 (nominal $P=0.0009$ ). Other ROA genes adding to the ES score (that is the genes that ranked before or at the point of maximize ES achieved, also denoted as leading-edge genes) are listed in Table 2 and Figure 1. Information of the representative SNPs for the
17 leading-edge genes that contributed positively to the ES is given in Table 3.

\section{Comparison analysis}

In the American whites, the ROA pathway was the only one that consistently presented the strongest association signals with height variation (nominal $P=0.002$, ES score $=0.55, Q_{\mathrm{FDR}}=0.042$, $P_{\mathrm{FWER}}=0.016$ ). The individual gene with the most significant nominal $P$-value is IFNA4 (nominal $P=0.0026$ ). Seven genes of the ROA pathway (IFNA4, IFNA21, PIK3C3, ULK2, IFNA14, IFNA13 and IFNG) closely associated with height in our GWAS with nominal $P$-value $<0.01$. The genes PIK3C3, ULK2 and IFNG presented significant nominal $P$-value $<0.01$ in both Han Chinese and American whites. The results from United States did not indicate other novel pathways or replicate the prior known ones regulating human height (all other tested pathways had $Q_{\mathrm{FDR}}>0.2$ and $P_{\mathrm{FWER}}>0.13$ ).

We also compared the $P$-values of the same representative SNPs in Chinese with in US whites, and none of them were significant (except for gene PRKAA2) (Table 4). Thus, different sample may have different representative SNPs even for the same gene, because of the different minor allele frequency and the distinct ethnic background.

\section{DISCUSSION}

The pathway-based approach can work as a complementary strategy to traditional SNP-based analyses and could be helpful for the

Table 2 Genes in the ROA pathway

\begin{tabular}{|c|c|c|c|}
\hline Gene symbol & Gene ID & Cytoband & Full name \\
\hline ATG12a & 9140 & $5 q 21-q 22$ & ATG12 autophagy related 12 homolog (S. cerevisiae) \\
\hline ATG3 & 64422 & $3 q 13.2$ & ATG3 autophagy related 3 homolog (S. cerevisiae) \\
\hline ATG4B & 23192 & $2 q 37.3$ & ATG4 autophagy related 4 homolog B (S. cerevisiae) \\
\hline ATG4Ca & 84938 & $1 \mathrm{p} 31.3$ & ATG4 autophagy related 4 homolog C (S. cerevisiae) \\
\hline ATG4D & 84971 & $19 p 13.2$ & ATG4 autophagy related 4 homolog D (S. cerevisiae) \\
\hline ATG5a & 9474 & $6 q 21$ & ATG5 autophagy related 5 homolog (S. cerevisiae) \\
\hline ATG7 ${ }^{a}$ & 10533 & 3p25.3-p25.2 & ATG7 autophagy related 7 homolog (S. cerevisiae) \\
\hline BECN1 & 8678 & $17 q 21$ & Beclin 1, autophagy related. (also know as ATG6 ) \\
\hline GABARAPL $1^{\text {a }}$ & 23710 & $12 \mathrm{p} 13.2$ & $\mathrm{GABA}(\mathrm{A})$ receptor-associated protein-like 1 \\
\hline GABARAPL2 & 11345 & $16 q 22.3-q 24$ & $\mathrm{GABA}(\mathrm{A})$ receptor-associated protein-like 2 \\
\hline IFNA1 & 3439 & $9 p 22$ & Interferon, $\alpha 1$ \\
\hline IFNA10 & 3446 & $9 p 22$ & Interferon, $\alpha 10$ \\
\hline IFNA13 ${ }^{a}$ & 3447 & $9 p 22$ & Interferon, $\alpha 13$ \\
\hline IFNA14a & 3448 & $9 p 22$ & Interferon, $\alpha 14$ \\
\hline IFNA16 ${ }^{a}$ & 3449 & $9 p 22$ & Interferon, $\alpha 16$ \\
\hline IFNA17 & 3451 & $9 p 22$ & Interferon, $\alpha 17$ \\
\hline IFNA2 & 3440 & $9 p 22$ & Interferon, $\alpha 2$ \\
\hline IFNA2 $1^{a}$ & 3452 & $9 \mathrm{p} 22$ & Interferon, $\alpha 21$ \\
\hline IFNA4 ${ }^{a}$ & 3441 & 9P22 & Interferon, $\alpha 4$ \\
\hline IFNA5 & 3442 & $9 \mathrm{p} 22$ & Interferon, $\alpha 5$ \\
\hline IFNA7 & 3444 & $9 \mathrm{p} 22$ & Interferon, $\alpha 7$ \\
\hline IFNA8 & 3445 & $9 p 22$ & Interferon, $\alpha 8$ \\
\hline IFNGa & 3458 & $12 q 14$ & Interferon, $\gamma$ \\
\hline PIK3C3a & 5289 & $18 q 12.3$ & Phosphoinositide-3-kinase, class3 (Vps34) \\
\hline PIK3R4 & 30849 & $3 q 22.1$ & Phosphoinositide-3-kinase, regulatory subunit 4 \\
\hline PRKAA1 & 5562 & $5 p 12$ & AMP-activated protein kinase $\alpha 1$ catalytic subunit \\
\hline PRKAA2 ${ }^{a}$ & 5563 & $1 \mathrm{p} 31$ & AMP-activated protein kinase $\alpha 2$ catalytic subunit \\
\hline ULK2 $2^{\mathrm{a}}$ & 9706 & $17 p 11.2$ & unc-51-like kinase \\
\hline
\end{tabular}

Abbreviations: ES, enrichment score; ROA, regulation-of-autophagy. ${ }^{a}$ Genes contributing positively to the ES score (leading-edge genes).

The first 10 genes encode critical proteins actively involved in the autophagy process.

The IFNA family and IFNG encode pleiotropic cytokines (type I and type II interferons), which regulate the autophagy process.

The Gene ID was retrieved from NCBI GenBank (http://www.ncbi.nlm.nih.gov/Genbank/). 
detection of novel mechanisms underlying complex diseases/phenotypes. In this study, this approach was used to analyze our genomic genotyping data for height variation in an elder Han Chinese population. ${ }^{13}$ The ROA pathway was suggested at the genome-wide level pathway-based approach after the multiple-testing correction. The result implied that autophagy-related biological processes may

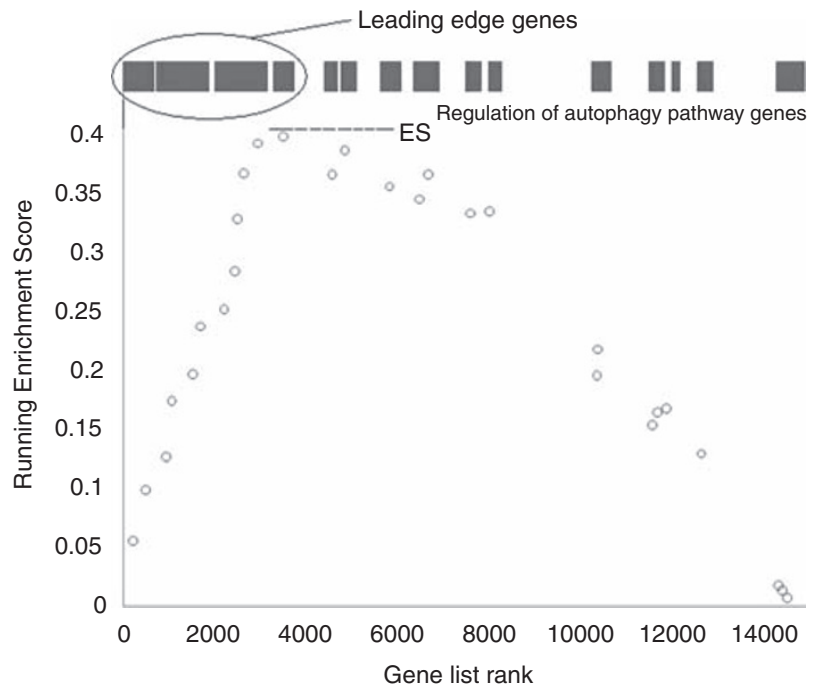

Figure 1 Running-sum plot for the ROA pathway, including the location of the maximum enrichment score (ES) and the leading-edge subset. The $x$ axis is the rank of the 28 genes in ROA pathway in the whole gene list generated. These 28 genes are, from left to right, PIK3C3, ULK2, ATG5, GABARAPL1, IFNG, ATG7, PRKAA2, IFNA10, IFNA7, IFNA14, IFNA21, ATG4C, IFNA4, IFNA5, ATG12, IFNA13, IFNA16, ATG3, IFNA2, ATG4D, IFNA8, ATG4B, PIK3R4, IFNA1, IFNA17, PRKAA1, GABARAPL2 and $B E C N 1$. The $y$ axis represents the running-enrichment score. The bar plot at the top of the figure also shows the distribution of the 28 genes from the ROA pathway at genome-wide level. have important roles in human stature variation, and thus may participate in the development and growth disorders of bones.

The ROA pathway (Pathway ID hsa04140 in KEGG database) is the unique pathway related to autophagy among all the pathways tested. Autophagy is a conservative house-keeping catabolic process that involves degradation of a cell's own components by formation and separation of a membrane around the targeted region of the cell. The resultant vesicle then fuses with a lysosome and then the inner contents degrade. The autophagy process was generally induced and enhanced by nutrient starvation, defense of infection or cell programmed death.

Involvement of the ROA pathway in human bone and cartilage growth has been demonstrated in earlier studies. Long bone growth and consequent increase in height take place through the activities of chondrocytes embedded in the epiphyseal growth plate. The embedded chondrocytes of long bones undergo autophagy and express autophagic proteins before the induction of osteogenesis. ${ }^{19}$ This is because autophagy enhances the survival of chondrocytes in the hypoxic microenvironments (the cartilage is avascular, thus contains little nutrition and oxygen) and induces the synthesis of the calcified extracellular matrix. ${ }^{20-22}$ In addition, the crosstalk of bone growth factors such as interleukin-3 with the ROA pathway regulates the utilization of exogenous nutrients to maintain cell viability. ${ }^{23}$ As a result, autophagy is an important intermediate stage during the terminal differentiation of chondrocytes and long bone development. Notably, the interferon- $\alpha$ family genes in the ROA pathway have some other effects on bone remodeling (Table 2). They can broadly suppress the proliferation of osteoprogenitor cells and inhibit the differentiation of monocytes to osteoclasts. ${ }^{24,25}$

Our study for the first time raises the importance of the ROA pathway to Chinese stature variation. The pathway was also highly suggested by our pathway-based genome-wide analysis that could influence the Caucasian height variation. In addition to highlighting its potential contribution to human height variation, the ROA pathway was demonstrated that associated with long bone mineral density at the ultra distal skeletal site (our unpublished data). Our findings in the bone mineral

Table 3 The representative SNPs mapped to the leading edge genes of the ROA pathway in Chinese and US whites

\begin{tabular}{|c|c|c|c|c|c|c|c|c|c|c|}
\hline Gene & SNPs in Chinese & Allele & Location & $M A F$ & $\begin{array}{c}\text { Nominal P-value } \\
\text { (Chinese) }\end{array}$ & $\begin{array}{l}\text { SNPS in US } \\
\text { whites }\end{array}$ & Allele & Location & $M A F$ & $\begin{array}{c}\text { Nominal P-value } \\
\text { (US Whites) }\end{array}$ \\
\hline PIK3C3 & rs2587613 & $\mathrm{C} / \mathrm{T}$ & 5'-Upstream & 0.12 & 0.0009 & rs16975296 & $A / G$ & 5'-Upstream & 0.07 & 0.0041 \\
\hline ULK2 & rs205096 & $\mathrm{C} / \mathrm{T}$ & Intronic & 0.30 & 0.0024 & rs17794370 & $A / G$ & Intronic & 0.27 & 0.0046 \\
\hline ATG5 & rs3827644 & $C / G$ & Intronic & 0.05 & 0.0053 & rs553307 & $A / G$ & 5'-Upstream & 0.08 & 0.0400 \\
\hline GABARAPL1 & rs11829144 & $\mathrm{C} / \mathrm{T}$ & Intronic & 0.06 & 0.0063 & rs10734824 & $A / G$ & 3'-Downstream & 0.02 & 0.3300 \\
\hline IFNG & rs2906852 & $\mathrm{C} / \mathrm{T}$ & 5'-Upstream & 0.16 & 0.0097 & rs1904559 & $\mathrm{C} / \mathrm{T}$ & 3'-Downstream & 0.12 & 0.0087 \\
\hline ATG7 & rs6442260 & $A / G$ & Intronic & 0.29 & 0.0112 & rs346081 & $\mathrm{C} / \mathrm{G}$ & Intronic & 0.17 & 0.0200 \\
\hline PRKAA2 & rs2796512 & $A / G$ & Intronic & 0.46 & 0.0162 & rs10889008 & $A / G$ & Intronic & 0.47 & 0.0400 \\
\hline IFNA10 & rs7849862 & $A / G$ & 5'-Upstream & 0.40 & 0.0186 & rs7849862 & $A / G$ & 3'-Downstream & 0.17 & 0.0100 \\
\hline IFNA7 & rs7856345 & $A / T$ & 3'-Downstream & 0.41 & 0.0194 & rs7849623 & $\mathrm{A} / \mathrm{T}$ & 3'-Downstream & 0.18 & 0.0100 \\
\hline IFNA14 & rs10811497 & $\mathrm{A} / \mathrm{T}$ & 3'-Downstream & 0.41 & 0.0209 & rs7037147 & $A / G$ & Intronic & 0.16 & 0.0061 \\
\hline IFNA21 & rs7047811 & $A / T$ & 5'-Upstream & 0.40 & 0.0246 & rs10964882 & $A / G$ & 5'-Upstream & 0.17 & 0.0034 \\
\hline ATG4C & rs1981067 & $\mathrm{C} / \mathrm{T}$ & Intronic & 0.30 & 0.0324 & rs11811517 & $\mathrm{A} / \mathrm{T}$ & Intronic & 0.23 & 0.0100 \\
\hline IFNA4 & rs7852323 & $\mathrm{C} / \mathrm{T}$ & 3'-Downstream & 0.41 & 0.0512 & rs7852323 & $\mathrm{A} / \mathrm{T}$ & 3'-Downstream & 0.17 & 0.0026 \\
\hline IFNA5 & rs10964938 & $\mathrm{G} / \mathrm{T}$ & 3'-Downstream & 0.34 & 0.0563 & rs10964938 & $\mathrm{A} / \mathrm{C}$ & 3'-Downstream & 0.18 & 0.0200 \\
\hline ATG12 & rs6883910 & $\mathrm{G} / \mathrm{T}$ & 5'-Upstream & 0.32 & 0.0800 & rs6883910 & $\mathrm{A} / \mathrm{C}$ & 5'-Upstream & 0.39 & 0.3100 \\
\hline IFNA13 & rs637949 & $C / G$ & 5'-Upstream & 0.27 & 0.0985 & rs637949 & $C / G$ & 5'-Upstream & 0.17 & 0.0064 \\
\hline IFNA16 & rs10811525 & $A / G$ & 5'-Upstream & 0.22 & 0.1043 & rs10811525 & $A / C$ & 3'-Downstream & 0.41 & 0.7200 \\
\hline
\end{tabular}

Abbreviations: MAF, minor allele frequency; ROA, regulation-of-autophagy; SNP, single-nucleotide polymorphism. 
Table 4 The comparison of $P$-values for same representative SNPs in Chinese and US whites

\begin{tabular}{|c|c|c|c|c|c|c|c|c|}
\hline Gene name & SNPs in Chinese & Allele & Physical position & Location & $\begin{array}{c}\text { MAF } \\
\text { (Chinese) }\end{array}$ & $\begin{array}{c}\text { MAF } \\
\text { (whites) }\end{array}$ & $\begin{array}{c}\text { Nominal P-value } \\
\text { (Chinese) }\end{array}$ & $\begin{array}{c}\text { Nominal P-value } \\
\text { (whites) }\end{array}$ \\
\hline PIK3C3 & rs2587613 & $\mathrm{C} / \mathrm{T}$ & chr18: 39164485 & 5'-Upstream & 0.12 & 0.32 & 0.0009 & 0.1052 \\
\hline ULK2 & rs205096 & $\mathrm{C} / \mathrm{T}$ & chr17: 19682327 & Intronic & 0.30 & 0.05 & 0.0024 & 0.5146 \\
\hline ATG5 & rs3827644 & $C / G$ & chr6: 106685195 & Intronic & 0.05 & 0.23 & 0.0053 & 0.1797 \\
\hline GABARAPL1 & rs1 1829144 & $\mathrm{C} / \mathrm{T}$ & chr12: 10350033 & Intronic & 0.08 & 0.06 & 0.0063 & 0.9644 \\
\hline IFNG & rs2906852 & $\mathrm{C} / \mathrm{T}$ & chr12:68379481 & 5'-Upstream & 0.16 & 0.24 & 0.0097 & 0.3415 \\
\hline ATG7 & rs6442260 & $A / G$ & chr3: 11590751 & Intronic & 0.29 & 0.35 & 0.0112 & 0.4654 \\
\hline PRKAA2 & rs2796512 & $A / G$ & chr1: 57149497 & Intronic & 0.47 & 0.44 & 0.0162 & 0.0220 \\
\hline IFNA10 & rs7849862 & $A / G$ & chr9: 21191684 & 5'-Upstream & 0.40 & 0.16 & 0.0186 & 0.2923 \\
\hline IFNA7 & rs7856345 & $A / T$ & chr9: 21196490 & 3'-Downstream & 0.41 & 0.18 & 0.0194 & 0.3437 \\
\hline IFNA14 & rs10811497 & $A / T$ & chr9: 21193432 & 3'-Downstream & 0.41 & 0.18 & 0.0209 & 0.4041 \\
\hline IFNA21 & rs7047811 & $A / T$ & chr9: 21181454 & 5'-Upstream & 0.40 & 0.18 & 0.0246 & 0.3507 \\
\hline ATG4C & rs1981067 & $\mathrm{C} / \mathrm{T}$ & chr1: 63322743 & Intronic & 0.30 & 0.46 & 0.0324 & 0.1463 \\
\hline IFNA4 & rs7852323 & $\mathrm{C} / \mathrm{T}$ & chr9: 21174643 & 3'-Downstream & 0.41 & 0.17 & 0.0512 & 0.1746 \\
\hline IFNA5 & rs10964938 & $\mathrm{G} / \mathrm{T}$ & chr9: 21258474 & 3'-Downstream & 0.34 & 0.17 & 0.0563 & 0.2559 \\
\hline ATG12 & rs6883910 & $\mathrm{G} / \mathrm{T}$ & chr5:115296082 & 5'-Upstream & 0.32 & 0.36 & 0.0800 & 0.3616 \\
\hline IFNA13 & rs637949 & $C / G$ & chr9: 21369315 & 5'-Upstream & 0.27 & 0.15 & 0.0985 & 0.3268 \\
\hline IFNA16 & rs10811525 & $A / G$ & chr9: 21288176 & 5'-Upstream & 0.22 & 0.43 & 0.1043 & 0.2450 \\
\hline
\end{tabular}

Abbreviations: MAF, minor allele frequency; SNP, single-nucleotide polymorphism.

The bold gene names indicated these eight genes cluster in a small region of chromosome 9 (also see Figure 2).

density study indicated that the ROA pathway may influence the height variation through different rate of long bone development and remodeling. Further functional researches are warranted.

A major strength of this study may be embodied by raised importance to height of the ROA pathway, which are largely neglected by previous GWASs. ${ }^{1}$ This may partially attribute to the pathwaybased approach applied here that allows to spontaneously examine the genetic information imbedded in multiple SNPs and genes. This nature of the pathway-based method alleviates the punishment for the multiple-testing problem, and allows examining the combined effects other than individual genes whose effects are too modest to be detected through single-SNP tests. Given the complexity of biological systems, such circumstance should not be rare. Therefore, we argue that the pathway-based tests can serve as a useful complement of traditional GWAS. In addition, we emphasized that as the pathway analysis aims to jointly consider multiple variants and measures the enrichment of gene set at the top of the entire ranked gene list, only a few dramatically significant SNPs in GWAS study (for example SNPs in IHH gene of Hedgehog Signaling) may not necessarily pull the entire pathway to the top. This phenomenon cannot be defined as the 'non-replication' for earlier studies.

However, several concerns need to be clarified in our study. First, the average age is over 70 years for Chinese and $>50$ years for Whites. To use stature data of aged group cannot represent a typical adult height variation. As the bone remodeling is active in the elder people, we may actually detect a pathway that enhanced the bone absorbing process. Some famous validated pathways (for example Hedgehog Signaling) in whites were not found by our study, one of the potential confounding factors could be the sample age bias. The ethnic difference was also another factor for non-replication because those pathways were initially identified at the Caucasian ethnic context. ${ }^{1}$

Second, our sample size is small. Large sample size can dramatically improve the statistical power for detecting the potential pathways. The association signal of ROA in our study (618 individuals) was weak (nominal $P=0.012$ ) compared with our US replication study (nominal
$P=0.002,1000$ individuals). Therefore, we reported the ROA pathway based on the statistical evidence with a consistently increased trend.

Third, although pathway-based analysis is a promising strategy to data mining of GWAS data sets, it is currently unclear what the best strategy is. Several methods are developed for pathway hunting recently. ${ }^{26-30}$ All of these methods used relatively complex model or simulation to improve the accuracy and statistical power. In fact, how to properly account for the gene size, gene number and linkage disequilibrium (LD) and how to adopt the optimal SNP weighting scheme (for example the representative SNPs located in the coding sequence could be more important than others) are worth further investigating. We used this method because it was generally robust to gene size, LD, gene number and, thus, accepted by the community. ${ }^{14,31,32}$ In addition, it was the real 'hypothesis-free' approach for pathway investigation, which means it can comprehensively test the association across different up-to-date pathway databases without prior hypotheses. Some studies first identified promising genes in one hypothesized pathway, then assessed the association of those genes with specific traits such as Parkinson disease. ${ }^{33,34}$ However, similar approaches were limited for quantitative complex traits (height), because the unknown fundamental biological processes may influence the phenotypes, such as the EphrinA-EphR pathway for bone geometry, ${ }^{31}$ the C-type natriuretic peptide signaling for human height. ${ }^{12}$

Finally, there are eight genes (eight representative SNPs) of ROA pathway cluster in a small region as shown in Figure 2 and Table 4. In addition, we constructed the LD block map for them (Figure 3), and six of them were located in one strong LD block. Although the permutation approaches may relieve this issue, ${ }^{14,32}$ the cluster of SNPs could still influence the result, and we lack a reliable method to accurately evaluate the extent of the influence. In our recent study, we performed the pathway-based study through shuffling the genotypic data (not phenotypic as the original). ${ }^{35}$ This procedure can completely eliminate the influence of strong $\mathrm{LD}$, because it breaks the structure of original LD. However, the simulated results showed that the falsepositive rate was not reduced; rather, it was slightly larger than the one by shuffling phenotypic data. Therefore, the influence of strong LD 


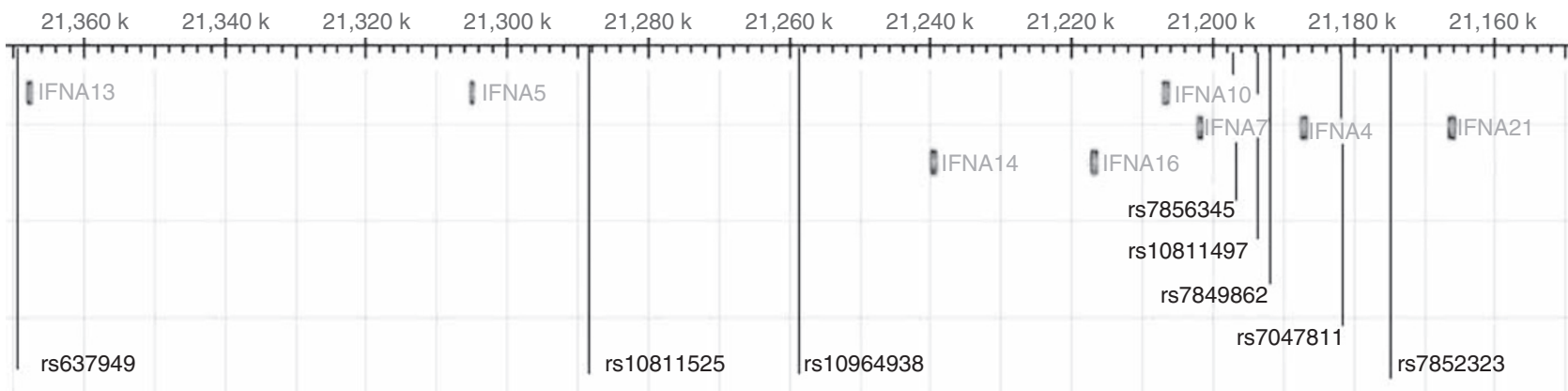

Figure 2 Physical position of the eight IFNA family members, including their corresponding representative SNPs located on the chromosome 9.
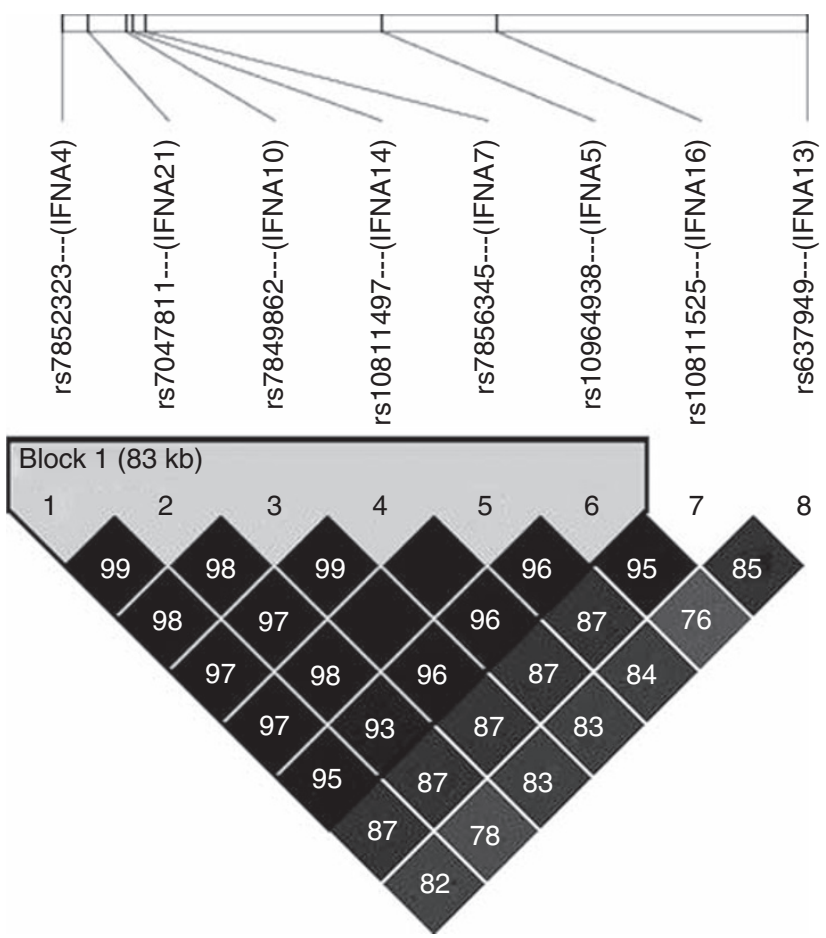

Figure 3 The haplotype block map for the eight representative SNPs of clustered IFNA family members. It was constructed using our own genotype data showing pair-wise linkage disequilibrium in $r^{2}$.

actually is far more less than one can expect. To permutate, the phenotypic data were a more conservative pathway-based association approach. To report this result, we mainly based on the statistical evidence and the independent replication study. Therefore, our study may only be served as a tentative investigation using this kind of test strategy. The future studies with larger sample size are necessary to validate our result.

In summary, the ROA pathway was suggestively associated with the height variation in Han Chinese in our pathway-based GWAS analyses. The genetic findings were supported by their biological implications in long bone development and replication study in the whites. Further functional researches are still warranted to fill the biological mechanism details.

\section{CONFLICT OF INTEREST}

The authors declare no conflict of interest.

\section{ACKNOWLEDGEMENTS}

We thank two anonymous reviewers for their time and effort in helping us improve and clarify this manuscript. HWD was partially supported by grants from NIH (P50AR055081, R01AG026564, R01AR050496, RC2DE020756, R01AR057049 and R03TW008221) and Franklin D Dickson/Missouri Endowment. The study also benefited from grants from National Science Foundation of China, Huo Ying Dong Education Foundation, HuNan Province, Xi'an Jiaotong University and the Ministry of Education of China. This study was supported by NIH grants from R01 AR050496, R21 AG027110, R01 AG026564, P50 AR055081 and R21 AA015973.

1 Weedon, M. N. \& Frayling, T. M. Reaching new heights: insights into the genetics of human stature. Trends Genet. 24, 595-603 (2008).

2 Li, M. X., Liu, P. Y., Li, Y. M., Qin, Y. J., Liu, Y. Z. \& Deng, H. W. A major gene model of adult height is suggested in Chinese. J. Hum. Genet. 49, 148-153 (2004).

3 Molho-Pessach, V., Lerer, I., Abeliovich, D., Agha, Z., Abu Libdeh, A., Broshtilova, V. et al. The $\mathrm{H}$ syndrome is caused by mutations in the nucleoside transporter hENT3. Am. J. Hum. Genet. 83, 529-534 (2008).

4 Ofluoglu, D., Unlu, F. \& Akyuz, G. Relationship between arm span and height in postmenopausal osteoporotic women. Rheumatol. Int. 28, 737-741 (2008).

5 Lei, S. F., Tan, L. J., Liu, X. G., Wang, L., Yan, H., Guo, Y. F. et al. Genome-wide association study identifies two novel loci containing FLNB and SBF2 genes underlying stature variation. Hum. Mol. Genet. 18, 1661-1669 (2009).

6 Lettre, G., Jackson, A. U., Gieger, C., Schumacher, F. R., Berndt, S. I., Sanna, S. et al. Identification of ten loci associated with height highlights new biological pathways in human growth. Nat. Genet. 40, 584-591 (2008).

7 Johansson, A., Marroni, F., Hayward, C., Franklin, C. S., Kirichenko, A. V., Jonasson, I. et al. Common variants in the JAZF1 gene associated with height identified by linkage and genome-wide association analysis. Hum. Mol. Genet. 18, 373-380 (2009).

8 Weedon, M. N., Lettre, G., Freathy, R. M., Lindgren, C. M., Voight, B. F., Perry, J. R. et al. A common variant of HMGA2 is associated with adult and childhood height in the general population. Nat. Genet. 39, 1245-1250 (2007).

9 Weedon, M. N., Lango, H., Lindgren, C. M., Wallace, C., Evans, D. M., Mangino, M. et al. Genome-wide association analysis identifies 20 loci that influence adult height. Nat. Genet. 40, 575-583 (2008).

10 Gudbjartsson, D. F., Walters, G. B., Thorleifsson, G., Stefansson, H., Halldorsson, B. V., Zusmanovich, P. et al. Many sequence variants affecting diversity of adult human height. Nat. Genet. 40, 609-615 (2008).

11 Krane, S. M. \& Inada, M. Matrix metalloproteinases and bone. Bone 43, 7-18 (2008). 12 Estrada, K., Krawczak, M., Schreiber, S., van Duijn, K., Stolk, L., van Meurs, J. B. et al. A genome-wide association study of northwestern Europeans involves the C-type natriuretic peptide signaling pathway in the etiology of human height variation. Hum. Mol. Genet. 18, 3516-3524 (2009).

13 Lei, S. F., Yang, T. L., Tan, L. J., Chen, X. D., Guo, Y., Guo, Y. F. et al. Genome-wide association scan for stature in Chinese: evidence for ethnic specific loci. Hum. Genet. 125, 1-9 (2009).

14 Wang, K., Li, M. \& Bucan, M. Pathway-based approaches for analysis of genomewide association studies. Am. J. Hum. Genet. 81, 1278-1283 (2007).

15 Pritchard, J. K., Stephens, M. \& Donnelly, P. Inference of population structure using multilocus genotype data. Genetics 155, 945-959 (2000).

16 Price, A. L., Patterson, N. J., Plenge, R. M., Weinblatt, M. E., Shadick, N. A. \& Reich, D. Principal components analysis corrects for stratification in genome-wide association studies. Nat. Genet. 38, 904-909 (2006).

17 Purcell, S., Neale, B., Todd-Brown, K., Thomas, L., Ferreira, M. A., Bender, D. et al. PLINK: a tool set for whole-genome association and population-based linkage analyses. Am. J. Hum. Genet. 81, 559-575 (2007).

18 Zheng, G., Freidlin, B., Li, Z. \& Gastwirth, J. L. Genomic control for association studies under various genetic models. Biometrics 61, 186-192 (2005). 
19 Srinivas, V. \& Shapiro, I. M. Chondrocytes embedded in the epiphyseal growth plates of long bones undergo autophagy prior to the induction of osteogenesis. Autophagy 2 215-216 (2006).

20 Srinivas, V., Bohensky, J., Zahm, A. M. \& Shapiro, I. M. Autophagy in mineralizing tissues: microenvironmental perspectives. Cell Cycle 8, 391-393 (2009).

21 Srinivas, V., Bohensky, J. \& Shapiro, I. M. Autophagy: a new phase in the maturation of growth plate chondrocytes is regulated by HIF, mTOR and AMP kinase. Cells Tissues Organs 189, 88-92 (2009).

22 Roach, H. I., Aigner, T. \& Kouri, J. B. Chondroptosis: a variant of apoptotic cell death in chondrocytes? Apoptosis 9, 265-277 (2004).

23 Lum, J. J., Bauer, D. E., Kong, M., Harris, M. H., Li, C., Lindsten, T. et al. Growth factor regulation of autophagy and cell survival in the absence of apoptosis. Cell 120, 237248 (2005).

24 Avnet, S., Cenni, E., Perut, F., Granchi, D., Brandi, M. L., Giunti, A. et al. Interferonalpha inhibits in vitro osteoclast differentiation and renal cell carcinoma-induced angiogenesis. Int. J. Oncol. 30, 469-476 (2007).

25 Oreffo, R. O., Romberg, S., Virdi, A. S., Joyner, C. J., Berven, S. \& Triffitt, J. T. Effects of interferon alpha on human osteoprogenitor cell growth and differentiation in vitro. J. Cell Biochem. 74, 372-385 (1999).

26 Herold, C., Steffens, M., Brockschmidt, F. F., Baur, M. P. \& Becker, T. INTERSNP: genome-wide interaction analysis guided by a priori information. Bioinformatics 25, 3275-3281 (2009).

27 O'Dushlaine, C., Kenny, E., Heron, E. A., Segurado, R., Gill, M., Morris, D. W. et al. The SNP ratio test: pathway analysis of genome-wide association datasets. Bioinformatics 25, 2762-2763 (2009).
28 Miller, D. J., Zhang, Y., Yu, G., Liu, Y., Chen, L., Langefeld, C. D. et al. An algorithm for learning maximum entropy probability models of disease risk that efficiently searches and sparingly encodes multilocus genomic interactions. Bioinformatics 25 , 2478-2485 (2009).

29 Torkamani, A. \& Schork, N. J. Pathway and network analysis with high-density allelic association data. Methods Mol. Biol. 563, 289-301 (2009).

30 Peng, G., Luo, L., Siu, H., Zhu, Y., Hu, P., Hong, S. et al. Gene and pathway-based second-wave analysis of genome-wide association studies. Eur. J. Hum. Genet. 18, 111-117 (2010).

31 Chen, Y., Xiong, D. H., Guo, Y. F., Pan, F., Zhou, Q., Zhang, F. et al. Pathway-based genome-wide association analysis identified the importance of EphrinA-EphR pathway for femoral neck bone geometry. Bone 46, 129-136 (2010).

32 Wang, K., Zhang, H., Kugathasan, S., Annese, V., Bradfield, J. P., Russell, R. K. et al. Diverse genome-wide association studies associate the IL12/IL23 pathway with Crohn Disease. Am. J. Hum. Genet. 84, 399-405 (2009).

33 Lesnick, T. G., Papapetropoulos, S., Mash, D. C., Ffrench-Mullen, J., Shehadeh, L., de Andrade, M. et al. A genomic pathway approach to a complex disease: axon guidance and Parkinson disease. PLoS Genet. 3, e98 (2007).

34 Lesnick, T. G., Sorenson, E. J., Ahlskog, J. E., Henley, J. R., Shehadeh, L., Papapetropoulos, S. et al. Beyond Parkinson disease: amyotrophic lateral sclerosis and the axon guidance pathway. PLoS One 3, e1449 (2008).

35 Guo, Y. F., Li, J., Chen, Y., Zhang, L. S. \& Deng, H. W. A new permutation strategy of pathway-based approach for genome-wide association study. BMC Bioinformatics 10, 429 (2009) 